\title{
Mixed Variant of Acrokeratosis verruciformis of Hopf: A Rare Entity
}

\author{
Sonia Jain ${ }^{1}$, Pratiksha Sonkusale ${ }^{1}$, Abhay Vilash Deshmukh², Pallavi Kumari ${ }^{1}$
}

${ }^{1}$ Department of Dermatology, Venereology \& Leprosy, ${ }^{2}$ Department of Pathology, Mahatma Gandhi Institute of Medical Sciences, Sevagram, Wardha, Maharashtra, India

\begin{abstract}
Acrokeratosis verruciformis of Hopf (AKV) is a rare autosomal dominant genodermatosis of unknown etiology. Here we present a case of a 20-year-female with multiple skin colored flat papules over dorsum of hands and feet interspersed with multiple hypopigmented macular lesion of 5 years duration. No family member showed similar lesion. Presence of classical AKV with absent family history and definite histopathology finding make this case an unusual and rare entity.

Key words: Acrokeratosis verruciformis of Hopf; genodermatosis; hypopigmented macules.
\end{abstract}

\section{Introduction}

A crokeratosis verruciformis of Hopf (AKV) is a rare autosomal dominant genodermatosis of unknown etiology. It is characterized by multiple flat topped, keratotic papules resembling plane warts, present mainly on the dorsum of hands and feet. It mostly presents in early childhood, but onset may be delayed as late as the fifth decade. ${ }^{1}$ It affects both the sexes with male to female ratio of $5: 1.3 .{ }^{2}$ Superficial ablation is the treatment of choice along with other treatment modalities including retinoic acid, cryotherapy and $\mathrm{CO}_{2}$ Iaser. $^{3}$

\section{Case report}

Twenty year old unmarried, female presented with multiple asymptomatic small firm skin colored eruption over dorsum of hands and feet since 5 years. Initially she noticed multiple small, discrete hypopigmented macules and skin colored papules over dorsum of hand and feet with gradual extension to bilateral forearms and legs (Figure1A and 1B). No family members had similar complaints. On examination, multiple discrete

Funding: No

Conflict of Interest: No

Address of Correspondence

Dr Sonia Jain

ORCID ID: 0000-0002-3376-2250

Department of Dermatology, Venereology \& Leprosy. Mahatma Gandhi Institute of Medical Sciences, Seagram, Wardha, Maharashtra, India, Pin code -442102

Tel: +91 9370868133, Fax: 07152-284333

E-mail: soniapjain1234@gmail.com skin to brown colored, firm, flat topped papules (approximately $0.5 \times 0.5 \mathrm{~cm}$ ) with multiple interspersed hypopigmented macules (approximately $0.5 \times 0.5 \mathrm{~cm}$ ) were present over bilateral forearm, hands, ankle and feet. No punctate keratosis on palms or nail involvement was present. Hair, oral mucosa and seborrheic areas of face and trunk were normal. No involvement of any other body parts was seen. Systemic examination was within normal limits. Routine blood investigations were also normal. With typical clinical findings, diagnosis of AKV was made. We kept the differential diagnosis of plane warts, seborrheic keratosis, epidermodysplasia verruciformis (EDV) and Darrier's disease. Skin biopsy showed hyperkeratosis, hypergranulosis, acanthosis, papillomatosis with circumscribed elevation of epidermis resembling "church spires" appearance with absence of parakeratosis and dyskeratosis (Figure 2A and $2 \mathrm{~B}$ ) confirmed the diagnosis of AKV. After taking proper consent, we got her liver enzymes and lipid profile tested. When her reports turned out normal, we started her on Isotretinoin $40 \mathrm{mg}$ in the dose of $1 \mathrm{mg} / \mathrm{kg} /$ body weight. Patient was called for follow

Date of Submission: $16^{\text {th }}$ June 2021

Date of Acceptance: $22^{\text {nd }}$ August 2021

Date of Publication: $1^{\text {st }}$ October 2021

How to cite this article

Jain S, Sonkusale P, Deshmukh AV, Kumari P. Mixed Variant of Acrokeratosis verruciformis of Hopf: A Rare Entity. NJDVL 2021;19(2):54-7. https://doi.org/10.3126/njdvl.v19i2.37813.

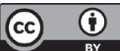

Licensed under CC BY 4.0 International License which permits use, distribution and reproduction in any medium, provided the original work is properly cited. 
up and clinical improvement was seen as flattening of skin colored papules with persistent hypopigmented macules after a period of around 6 weeks (Figure $3 \mathrm{~A}$ and $3 \mathrm{~B})$. However, we had to discontinue the treatment after 8 weeks as patient complained of amenorrhoea. We were also unable to explain presence of hypopigmented macules since they remain unchanged.

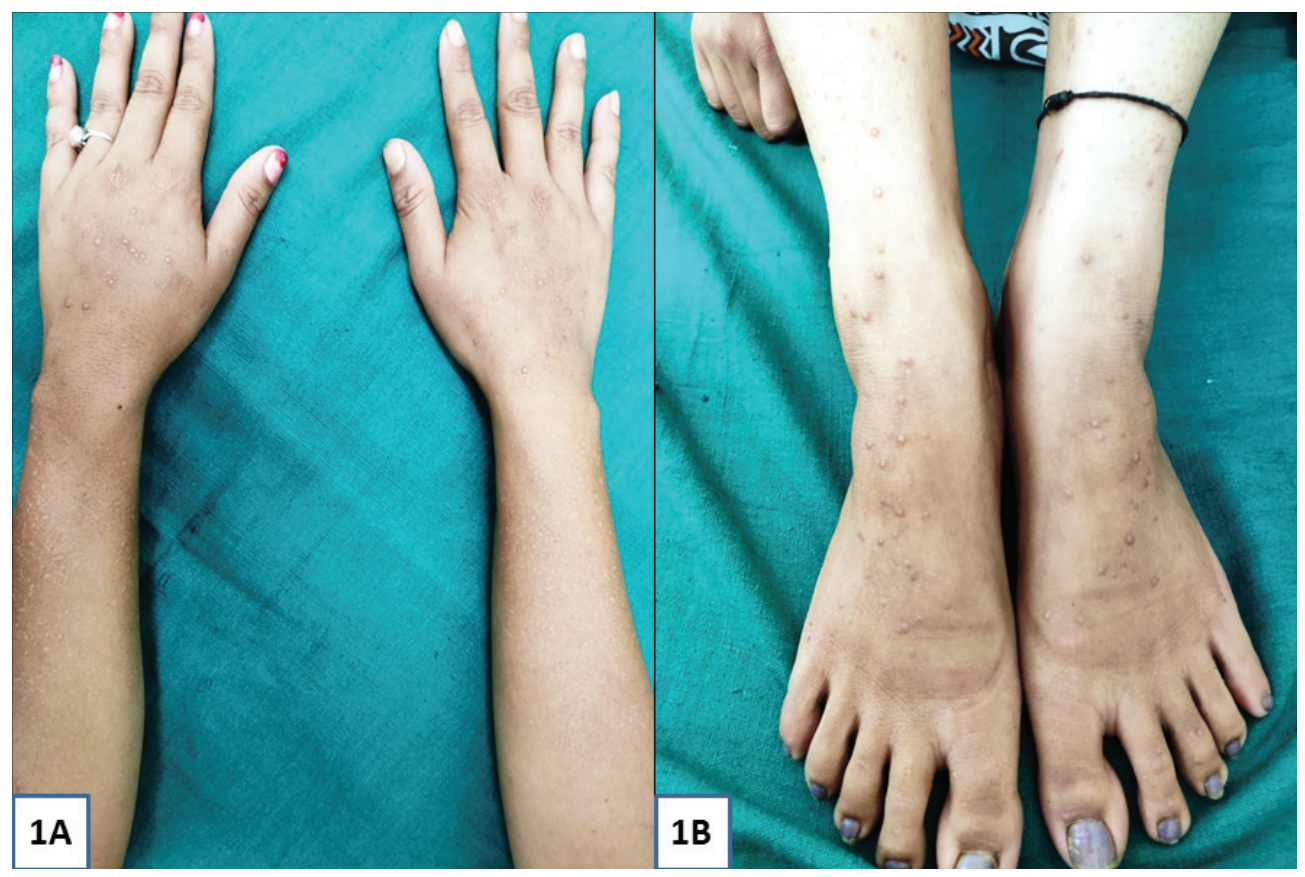

Figure 1A and 1B: Multiple discrete skin to brown colored, firm, flat-topped papules with interspersed hypopigmented macules over bilateral forearm, hands, ankle and feet.

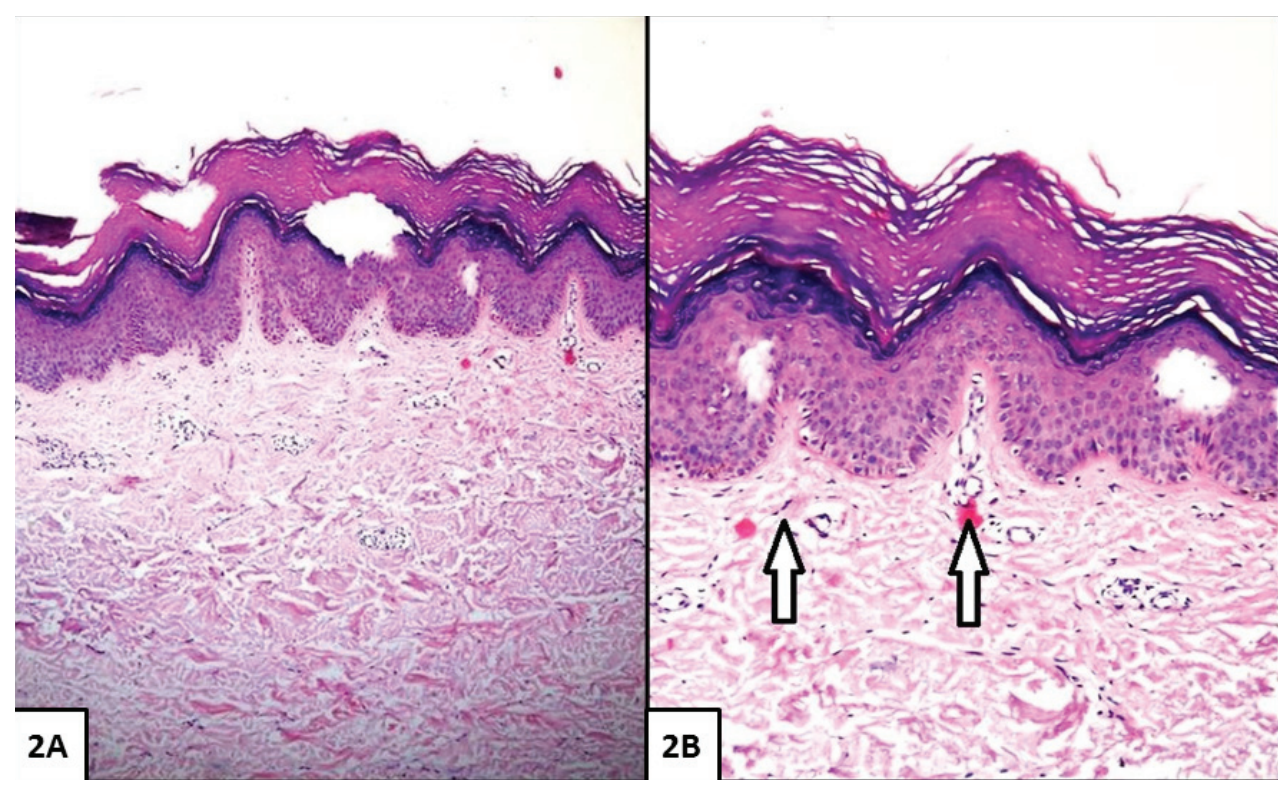

Figure 2A and 2B: Section shows hyperkeratosis, hypergranulosis, papillomatosis with circumscribed elevation of epidermis resembling "church spire" appearance (arrow) in 100X and 400X. 


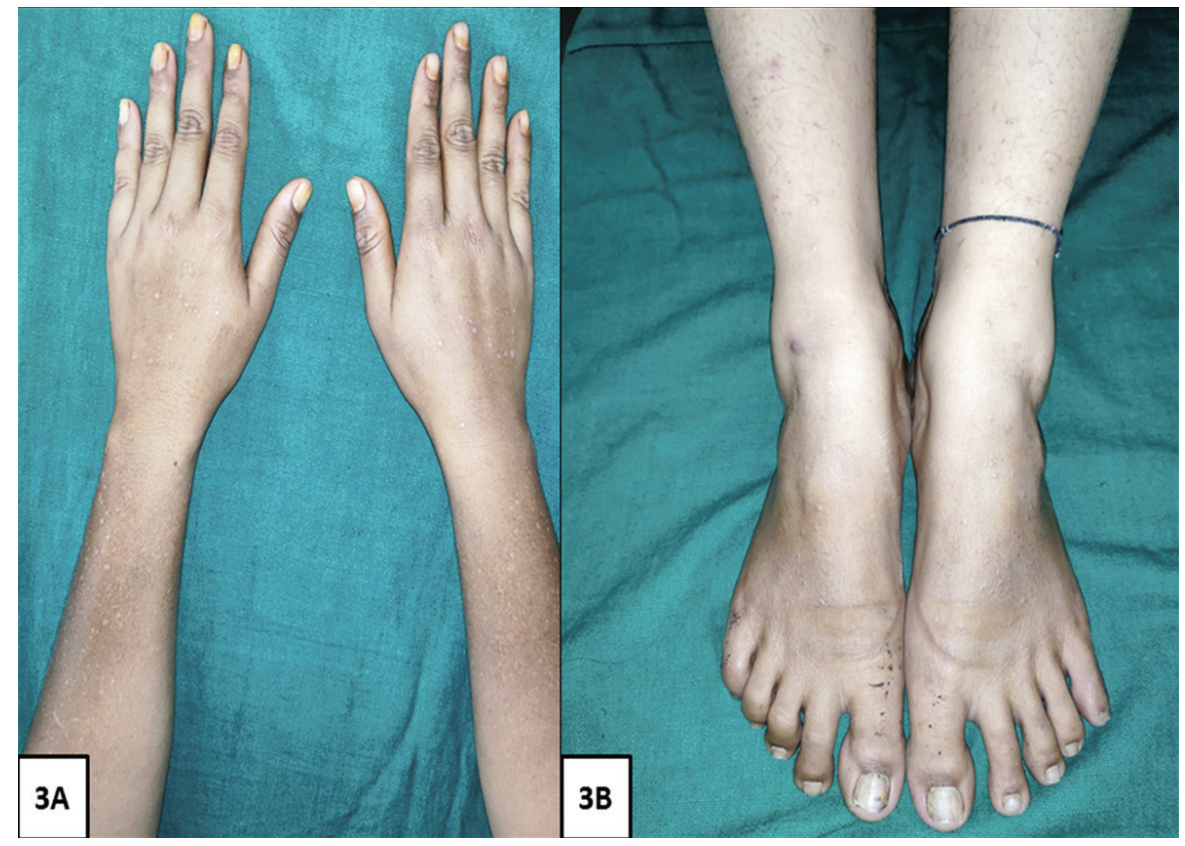

Figure 3A and 3B: Post-treatment images with flattening of skin colored papules and persistent hypopigmented macules over bilateral forearm, hands, ankle and feet.

\section{Discussion}

AKV was first described by Hopf in $1931 .^{1}$ The probable etiology is a mutation in the ATP2A2 gene located on chromosome 12q24, a heterozygous pro602Leu mutation, the same gene affected in Darier disease (DD). ${ }^{4}$ This mutation has an autosomal dominant pattern of inheritance but incomplete penetrance so family history may not be always present. ${ }^{1}$ Since this is a dominant mutation, the disease follows a chronic course without remission. ${ }^{1}$ The lesions can rarely undergo malignant transformation to squamous cell carcinoma. ${ }^{1}$

Clinically, AKV often occurs in childhood as multiple reddish-brown or skin colored, flat-topped papules mainly on the dorsum of the hands and feet and may involve knees, elbows, forearms or lower legs. The palmar skin may be thickened with punctate keratosis or punctiform breaks in dermatoglyphics. Nail changes involve longitudinal splitting, striations and subungual hyperkeratosis. ${ }^{5}$

Classical AKV often occurs during childhood with typical morphology on the dorsum of hands and feet. Sporadic AKV has late age of onset and may affect other sites like face, scalp and trunk. Positive family history with nail changes and palmar pits are seen in classical AKV but not in sporadic AKV. ${ }^{6}$ Bang et al. found reported six sporadic AKV cases having typical clinical and histological finding with no positive family history. ${ }^{6}$ This may be due to incomplete penetrance of the mutated gene. He noticed differences between familial AKV and non-familial AKV. In classical AKV, Dhitavat et al. ${ }^{7}$ have reported novel P602 L mutation within the ATP binding domain of ATP2A 2 and Berk et al. ${ }^{8}$ reported an A698V codon change in ATP2A2 in sporadic AKV. In our case, onset was in late adolescence with involvement of dorsum of hand and feet suggestive of Classical AKV but without involvement of nails and palms and an absent family history supporting sporadic AKV.

The differential diagnosis plane wart, seborrheic keratosis, Darier's disease and EDV clinically mimic the lesions of AKV but are distinguished on histologic features. Lesions can be cytologically evaluated to rule out epidermodysplasia verruciformis. A family history of similar lesion can be evaluated to determine if there is any genetic component of Darier and AKV more likely and plane wart and seborrheic keratosis less likely. ${ }^{1,2}$ Ultimately, a biopsy and histological evaluation will be beneficial in diagnosing this condition. Histopathologically, AKV shows hyperkeratosis, hypergranulosis, acanthosis, papillomatosis with circumscribed elevation of epidermis resembling "church spires" appearance with some degree of acantholysis, absence of pakeratosis, dyskeratosis or basal layer change which is present in Darier's disease. ${ }^{9}$ Hyperkeratosis, hypergranulosis with vacuolation and koiliocytes with mild to moderate dysplasia in the epidermis in EDV while the absence of squamous and basaloid cells distinguish seborrheic keratosis. Though biopsy is gold standard, Dermoscopy is also valuable to differentiate these clinical entities (from verruca plana and seborrheic keratosis). Dermoscopy of AKV show 
white homogenous areas, central white network, peripheral cobble stone appearance with typical "Sunray appearance" and regularly arranged brown dots in the non-lesional skin. ${ }^{10,11}$

The effective treatment option for AKV is superficial ablation. $^{3}$ Other options include topical retinoic acid, cryotherapy or destructive lasers such as $\mathrm{CO}_{2}$ or $\mathrm{Nd}: Y A G{ }^{3}$ Oral retinoids showed variable results with few cases which have been successfully treated with oral isotretinoin. ${ }^{12}$

Our case is unique with mixed variant of AKV in a female gender with absent family history, no palmar pits or nail changes associated with hypopigmented macules. She was treated successfully with oral

\section{References}

1. Andrade TC, Silva GV, Silva TM, Pinto AC, Nunes AJ, Martelli AC. Acrokeratosis verruciformis of Hopf - Case report. An Bras Dermatol. 2016 Sep-Oct;91(5):639-641. doi: 10.1590/abd18064841.20164919

2. Rege VL, Hede R, Nadkarni NS. Acrokeratosis verruciformis of Hopf. Indian J Dermatol Venereol Leprol 1992;58:95-8. https://ijdvl.com/ acrokeratosis-verruciformis-of-hopf-2.

3. Nair PA. Acrokeratosis verruciformis of Hopf along lines of Blaschko. Indian J Dermatol. 2013; 58: 406406. doi: 10.4103/0019-5154.117324

4. Harman M, Durdu M, Ibiloglu I. Acrokeratosisverruciformis of Hopf exhibiting Darier disease-like cytological features. Clin. Exp. Dermatol. 2016 Oct; 41(7):761-3. doi: 10.1111/ ced.12910

5. Rallis E, Economidi A, Papadakis P, Verros C. Acrokeratosis verruciformis of Hopf (Hopf disease): case report and review of the literature. Dermatol Online J. 2005 Aug 1;11(2):10. https://pubmed. ncbi.nlm.nih.gov/16150218

6. Bang $\mathrm{CH}$, Kim HS, Park YM, Kim HO, Lee JY. Nonfamilial acrokeratosis verruciformis of Hopf. Ann Dermatol 2011;23 Suppl 1:S61-3. doi: 10.5021/ ad.2011.23.S1.S61

7. Dhitavat J, Macfarlane S, Dode L, Leslie N, Sakuntabhai A, MacSween R, et al. Acrokeratosis verruciformis of Hopf is caused by mutation in ATP2A2: evidence that it is allelic to Darier's disease. J Invest Dermatol. 2003 Feb;120(2):22932. doi: 10.1046/j.1523-1747.2003.t01-1-12045.x.

8. Berk DR, Taube JM, Bruckner AL, Lane AT. A sporadic patient with acrokeratosis verruciformis of isotretionoin. Damarla SV et al. reported AKV with hypopigmentation in family. ${ }^{13}$ This may help to highlight AKV with hypogimented macules, which could be a variant, to categorize genodermatosis. Moreover, AKV and Darier's disease are considered allelic disorder, the probable cause of hypopigmented macules can be defective keratinization interfering with melanosome transfer and an overall disruption of the "epidermal melanin unit" which was reported by Sornakumar and Srinivas in their case of Darier's with perifollicular hypopigmentation. ${ }^{14}$ Macular variant of AKV with absent family history was reported by Vora RV et al. ${ }^{15}$ Also, in the treatment of AKV oral isotretinoin could be considered as an effective treatment modality if superficial ablation and laser therapy are not available.

Hopf and a novel ATP2A2 mutation. Br J Dermatol. 2010 Sep;163(3):653-4. doi: 10.1111/j.13652133.2010.09876.x

9. Johnson BL Jr, Honig P. Congenital diseases (genodermatosis). In: Lever's Histopathology of the Skin. $9^{\text {th }}$ ed. Philadelphia: JB Lippincott; 2005. p. 156-9. https://scholar.google.com/scholar_lookup ?title=Lever\%27s+Histopathology+of+the+Skin\&p ublication_year=2005

10. Behera B, Prabhakaran N, Naveed S, Kumari R, Thappa DM, Gochhait D. Dermoscopy of acrokeratosis verruciformis of Hopf. J Am Acad Dermatol. 2017 Aug;77(2):e33-e35. doi: 10.1016/j. jaad.2017.01.003

11. Rajan M B, Bains A, Vedant D. Dermoscopy of acrokeratosis verruciformis of hopf. Indian Dermatol Online J 2021;12:374-5. doi: 10.4103/ idoj.IDOJ_598_19

12. Sharma R K, Vyas S. A Sporadic Case of Darier Disease with Acrokeratosis Verruciformis like Lesions: Treated Effectively with Oral and Topical Retinoid. International Journal of Innovative Research in Medical Science, 2017;2 :03, 599-601. https://doi.org/10.23958/ijirms/vol02-i03/02

13. Damarla SV, Arakkal GK, Chintagunta SR, Vasavi Latha C H. Acrokeratosis verruciformis: An unusual presentation. J NTR Univ Health Sci 2016;5:3.3-5. doi: 10.4103/2277-8632.196596

14. Sornakumar L, Srinivas C R. Darier's disease with perifollicular hypopigmentation. Indian J Dermatol 2010;55:299-300. doi: 10.4103/0019-5154.70685

15. Vora RV, Diwan NG, Jivani NB, Singhal RR, Gandhi SS. Macular variant of acrokeratosis verruciformis of Hopf. Med J DY Patil Univ 2017;10:195-7. doi: 10.4103/0975-2870.202096 